\title{
Gravitational Blue Shift Confirms the New Phenomenon of the Vertical Aether Flow into any Mass
}

\author{
M. E. Isma'eel*, Sherif M. E. Ismaeel \\ Formerly; Deputy Manager of the Armed Forces Technical Research Center of Egypt \\ Dr. Sherif is a lecturer with Ain Shams University Faculty of Science, Department of Physics
}

\begin{abstract}
In fact, the vertical position of Michelson-Morley experiment is not the only possible explanation for the new phenomenon of the vertical Aether flow into any mass or any fundamental building block. This paper shows, for the first time, that the cosmic blue shift due to a gravitational field is a direct consequence of the vertical Aether flow into any mass. The vertical Aether speeds of different stellar objects have been given, which suggest reclassifying the categories of black holes. To confirm the theory presented, new formulas for Doppler Effect in a gravitational field and its correlation with the time dilation, as derived from the General Relativity, has also been derived. The theoretical expressions corresponding to the two experimental results have been given. Also, a new prediction has been proposed, for the first time, to confirm the theories presented in this paper.
\end{abstract}

\section{Introduction}

So far, unfortunately the scientific community is still reluctant to accept the idea of the existence of the Aether as the medium pervading (or permeating) all the space and it flows vertically to the surface of any mass including any elementary particle. At present, in additions to the theories introduced in reference [1], there are decisive experiments carried out in both US and Germany which proves the existence of the vertical Aether flow. Back in history, Albert Einstein discarded the theory of Aether existence in 1905 of the last century - in his Special Theory of Relativity - after Michelson and Morley had failed to prove its existence in their famous (horizontal) experiment carried out in 1887. Einstein and - may be - the majority of scientists believe that the length contraction, $l=l_{o} \sqrt{1-\frac{v^{2}}{c^{2}}}$, where $v$ is the Earth speed in its orbit around the sun, is the phenomenon responsible for the null result of Michelson-Morley experiment. Thus they are ignoring other tremendous speeds of Earth's motion shown in table I.

Table I: Hierarchy of the Earth Motion in the Continuum Aether

\begin{tabular}{|c|c|c|}
\hline$\#$ & Types of Motion & Speed \\
\hline 1 & The peripheral velocity of the Earth surface due to its spinning about its axis (the Daily Rotation) & $\begin{array}{c}0.5 \mathrm{Km} / \mathrm{sec} . \\
\text { (at the Equator) }\end{array}$ \\
\hline 2 & The average Orbital velocity of the Earth around the Sun & $30 \mathrm{Km} / \mathrm{sec}$. \\
\hline 3 & $\begin{array}{c}\text { The velocity of the Sun, and hence the Earth, with respect to the local field of stars in the direction } \\
\text { of an imaginary point in the constellation Hercules, near the bright star Vega. }\end{array}$ & $19.7 \mathrm{Km} / \mathrm{sec.}$ \\
\hline 4 & The velocity of the Solar System, and hence the Earth, around the bulge of our Milky Way Galaxy & $217-250 \mathrm{Km} / \mathrm{sec}$. \\
\hline 5 & $\begin{array}{c}\text { The velocity of our Milky Way Galaxy as a member in the moving Local Group of galaxies in the } \\
\text { direction of the constellation Hydra. These measurements, confirmed by the Cosmic Background } \\
\text { Explorer satellite in 1989 and 1990. }\end{array}$ & $600 \mathrm{Km} / \mathrm{sec.}$ \\
\hline 6 & $\begin{array}{c}\text { The velocity of the Local Group as a member of the Local Super cluster (Virgo Super Cluster), in } \\
\text { the direction of the Norma cluster (Abell 3627) }\end{array}$ & $600 \mathrm{Km} / \mathrm{sec}$. \\
\hline 7 & $\begin{array}{c}\text { Our Milky Way galaxy being pulled towards a much more massive cluster of galaxies near the } \\
\text { Shapley Super-Cluster which lies beyond the Great Attractor (A localized concentration of mass } \\
\text { equivalent to tens of thousands of Milky Way's). }\end{array}$ & $700 \mathrm{Km} / \mathrm{sec.}$ \\
\hline 8 & $\begin{array}{c}\text { According to Hubble's Law, the Average velocity of our Milky Way Galaxy in the Accelerating } \\
\text { Expanding Universe as located in an intermediate distance between the center of explosion of the } \\
\text { universe (a hypothetical point) and the edge (the event horizon) of the universe. }\end{array}$ & $150,000 \mathrm{Km} / \mathrm{sec}$. \\
\hline
\end{tabular}

Now, despite of the fact that Michelson and Morley carried out their experiment on the bases of the Earth's motion only around the sun (due to the lack of knowledge and data at their time), the expected interference pattern of Michelson-Morley interferometer must record and include all the effects of all other types of Earth's motions (in the continuum Aether) shown in table I. Of course, this is directly followed as nature acts quite independent of Michelson and Morley understanding as well as our understanding of the story too! Now, as we get null results of this experiment, despite of the existence of these numerous and tremendous types of 
earth's motions, then we can easily conclude that Michelson-Morley experiment has proved, beyond any shadow of a doubt and with a lot of confidence, that either Aether does not exist, or Aether is flowing vertically to the horizontal plane of the interferometer, which is the truth. It should be noted that, any change in the data of table I (or even change in its accuracy) does not alter the bases of the principle used here, as the earth still has numerous types of motions in the absolute space, i.e. in the Continuum Aether.

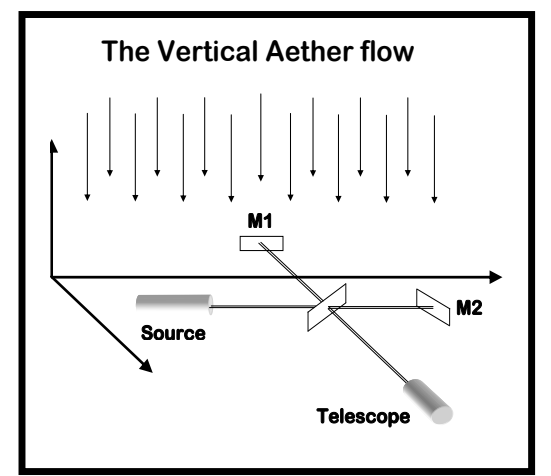

Figure a: Shows the typical horizontal plane of Michelson-Morley Interferometer, which is normal to the Aether flow. No interference pattern is observed.

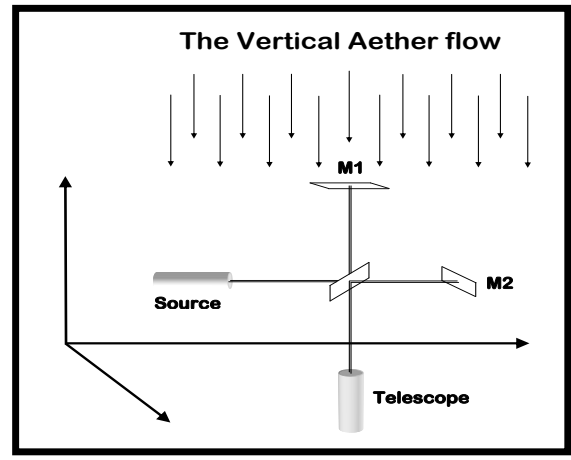

Figure b: Shows the new vertical plane of Michelson-Morley Interferometer, where the interference pattern can be observed.

Finally, the broad sense of this phenomenon indicates that, not only Aether flows vertically onto earth's surface, but also it flows vertically to the surfaces of planets, stars, black holes and any stellar object as well as any elementary particle in Nature. Thus in order to prove that the Aether exists, Michelson-Morley interferometer should be put in a vertical plane instead of the common horizontal plane as shown in figures a and $b$.

\section{Doppler Effect in a gravitational Field and the Aether Speed}

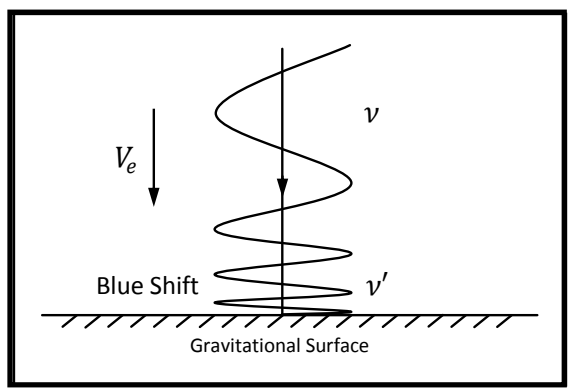

Figure c: The gravitational blue shift of a light wave as it moves downward in a gravitational field of mass $M$.

Let us assume a wave of frequency $v$ approaching any stellar object of mass $M$, and its frequency at the surface of the object is changed to $v^{\prime}$, as shown in figure c. Then, we can write the following energy equation;

$$
h v^{\prime}=h v+m g L
$$

Where $h$ is Planck's constant, $m$ is the photon mass, $g$ is the gravitational acceleration of the mass $M$ and $L$ is the falling length. With

$$
m c^{2}=h v, \text { then } m=h v / c^{2}
$$

Also, the gravitational acceleration of the mass $M$ at any distance $r$ from its center of mass is directly followed from Newton's gravitational law by;

Substitute equations (2) and (3) into (1), we get

$$
g=-G M / r^{2}
$$

$$
h v^{\prime}=h v+\frac{h v}{c^{2}}\left(-\frac{G M}{r^{2}}\right) L
$$


Let the change in frequency $\left(v^{\prime}=v+\delta v\right)$ of the wave when it falls a distance $L=\delta r$. Then equation (4) will take the form,

Which yields;

$$
h(v+\delta v)=h v+\frac{h v}{c^{2}}\left(-\frac{G M}{r^{2}}\right) \delta r
$$

$$
\frac{\delta v}{v}=-\frac{M G}{c^{2}} \frac{\delta r}{r^{2}}
$$

Equation (6) can be integrated over the falling length to get,

After minor manipulation, we can get

$$
\int_{v}^{v^{\prime}} \frac{\delta v}{v}=-\frac{M G}{c^{2}} \int_{r}^{r^{\prime}} \frac{\delta r}{r^{2}}
$$

$$
v^{\prime}=v e^{\frac{M G}{c^{2}}\left(\frac{1}{r^{\prime}}-\frac{1}{r}\right)} \quad r^{\prime}<r
$$

Equation (7) is the Doppler Effect in a gravitational field. It gives the blue shift of the frequency as the wave approaches a gravitational mass $M$. The red shift is obtained by rewriting equation (7) in the following form;

$$
v^{\prime}=v e^{-\frac{M G}{c^{2}}\left(\frac{1}{r}-\frac{1}{r^{\prime}}\right)} \quad r<r^{\prime}
$$

Now let us postulate that the change in the frequency of the wave, $\delta v$, is directly proportional to the change, $\delta V_{e}$ , of the velocity of the vertical Aether flow into the mass $M$. Then we can write,

$$
\delta v \propto \delta V_{e}
$$

Let us accept, for the moment, the following relationship;

$$
\frac{\delta v}{v}=\frac{\delta V_{e}}{4 c}
$$

Where $c$ is the speed of light. This means that the per unit change in frequency is equal one forth the change in the Aether - vertical - speed with respect to the speed of light. On integrating equation (9),

Then we get,

$$
\int_{v}^{v^{\prime}} \frac{\delta v}{v}=\frac{1}{4 c} \int_{V_{e}}^{V_{e}^{\prime}} \delta V_{e}
$$

$$
v^{\prime}=v e^{\frac{1}{4 c}\left(V_{e}^{\prime}-V_{e}\right)}
$$

Equation (10) is the Doppler Effect in a gravitational field in terms of the Aether speed. Compare equations (7) and (10), we get the following relation

$$
\frac{V_{e}}{4 c}=\frac{M G}{r c^{2}}
$$

Which yields the vertical Aether speed into any gravitational mass $M$,

$$
V_{e}=\frac{4 M G}{r c}
$$

Equation (11) is the same equation as the one derived from the general relativity, as we shall see in the next section.

\section{Grazing Angle Derived from the General Relativity}

To confirm our point of view, let us go back to the early of the year 1911, when Albert Einstein directly addressed the influence of gravity on light ("Über den Einfluß der Schwerkraft auf die Ausbreitung des Lichtes" / "On the Influence of Gravity on the Propagation of Light"). At this time, the General Theory of Relativity was not fully developed. This is the reason why Einstein obtained - unaware of the earlier result - the same value for the deflection angle as had been calculated with Newtonian physics, and Einstein found that,

$$
\alpha_{\text {Newton }}=\frac{2 G M}{c^{2} r}
$$

for the deflection angle of a ray grazing the sun at a distance $r$ from its center. In this relation $M$ and $r$ are the mass and the radius of the sun; $c$ and $G$ are the velocity of light and the gravitational constant respectively.

With the completion of the General Theory of Relativity in 1916, Einstein was the first to derive the correct deflection angle $\alpha$ of a light ray passing at a distance $r$ from an object of mass $M$ as

$$
\alpha=\frac{4 G M}{c^{2} r}
$$

Which is exactly twice the value derived from Newton's theory of gravity. The additional factor of two (compared to the "Newtonian" value) reflects the spatial curvature, which is missed if photons are just treated as particles. To confirm the truthfulness of this equation, we use the following numerical values, with radius of the sun; $r=6.96 \times 10^{8}$ meter, its mass, $M=1.9891 \times 10^{30} \mathrm{~kg}, G=6.67300 \times 10^{-11} \mathrm{~meter}^{3} \mathrm{~kg}^{-1} \mathrm{~s}^{-2}$, and $c=3 \times 10^{8} \mathrm{~meter} / \mathrm{s}$, Einstein obtained his famous grazing angle; 


$$
\alpha=1.74 \operatorname{arcsec}
$$

It is known that this value was measured within accuracy $20 \%$ during the solar eclipse in 1919 determined by Arthur Eddington and his group. This grazing angle was the second observational confirmation of the General Theory of Relativity and the basis of Einstein's huge popularity starting in the 1920's. The first confirmation of the general theory of relativity was the explanation of Mercury's perihelion shift. Recently, the value of $\alpha$ predicted by Einstein was confirmed to accuracy better than $0.02 \%$.

\section{Aether Speed; based on the General Relativity}

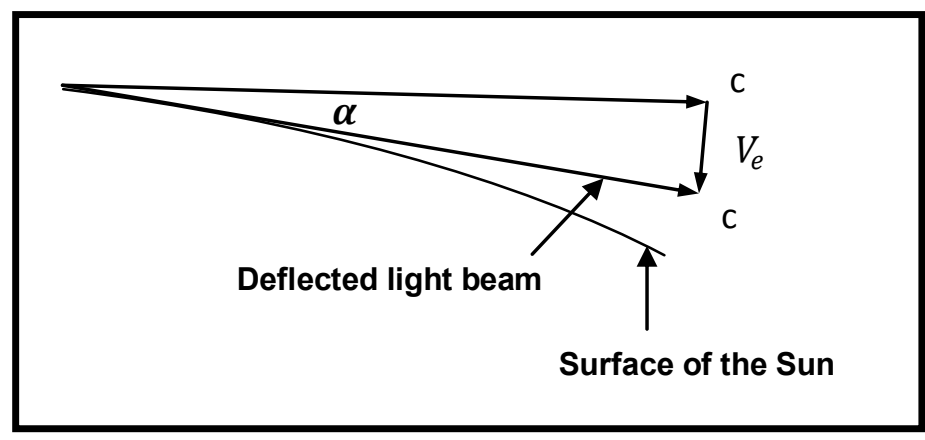

Figure d: A deflected light beam near the Surface of the Sun (or any star).

Now, by considering the grazing angle of the light beam is a direct effect of the vertical flow of the Aether to the surface of the sun, as shown in figure d. Then from the shown figure, the normal speed of Aether, $V_{e}$, is given approximately by the following formula;

Or

$$
V_{e}=c \times 2 \sin (\alpha / 2) \approx c \times 2 \times \alpha / 2=c \alpha
$$

Where $\alpha$ is the grazing angle.

$$
\alpha=V_{e} / c
$$

It is clear from equation (16) that the grazing angle $\alpha$ is the speed ratio between the vertical Aether speed $V_{e}$ and the light speed $c$. Eliminate $\alpha$ between equations (13) and (16), then, the vertical Aether speed will be given by;

Which is equation (11) derived before.

$$
V_{e}=\frac{4 G M}{c r}
$$

Table II: Shows the Vertical Aether Speed of Different Stellar Masses

\begin{tabular}{|c|c|c|c|}
\hline Class & Mass & Radius & Vertical Aether Speed \\
\hline Moon & $7.3477 \times 10^{22} \mathrm{Kg}$ & $1.738 \times 10^{6} \mathrm{~m}$ & $0.037576 \mathrm{~m} / \mathrm{s}$ \\
\hline Earth & $5.9742 \times 10^{24} \mathrm{Kg}$ & $6.3781 \times 10^{6} \mathrm{~m}$ & $0.83339 \mathrm{~m} / \mathrm{s}$ \\
\hline Sun & $1.9891 \times 10^{30} \mathrm{~kg}$ & $6.96 \times 10^{8} \mathrm{~m}$ & $2.535485 \times 10^{3} \mathrm{~m} / \mathrm{s}$ \\
\hline Super-massive black hole & $\sim 10^{5}-10^{9} \mathrm{M}_{\text {Sun }}$ & $\sim 0.001-10 \mathrm{AU} *$ & $3.932819 c \mathrm{~m} / \mathrm{s}$ \\
\hline Intermediate-mass black hole & $\sim 10^{3} \mathrm{M}_{\text {Sun }}$ & $\sim 10^{3} \mathrm{~km}$ & $5.89923 c \mathrm{~m} / \mathrm{s}$ \\
\hline Stellar-mass & $\sim 10 \mathrm{M}_{\text {Sun }}$ & $\sim 30 \mathrm{~km}$ & $1.96641 \boldsymbol{c} \mathrm{m} / \mathrm{s}$ \\
\hline Micro black hole & up to $\sim \mathrm{M}_{\text {Moon }}$ & up to $\sim 0.1 \mathrm{~mm}$ & $2.176881 \boldsymbol{c} \mathrm{m} / \mathrm{s}$ \\
\hline
\end{tabular}

\section{* $\mathrm{AU}$ is the astronomical unit $\left(\sim 150 \times 10^{6}\right.$ kilometers $), c$ is the speed of light}

Black holes are commonly classified according to their masses, independent of their angular momenta. Noting that, the size of a black hole, is determined by the radius of the event horizon, or by Schwarzschild radius, $r_{s}=2 G M / c^{2}$.

As seen from table II, the vertical speed of the Aether on Earth surface is about $83.4 \mathrm{~cm} / \mathrm{sec}$., while that on the Sun surface is about $2.54 \mathrm{~km} / \mathrm{sec}$. Also as seen from the table, the Aether speed on the surface of the Intermediate-mass black hole is $(5.89923 \boldsymbol{c ~ m} / \mathrm{s})$, where $\boldsymbol{c}$ is the speed of light, which is greater than that of the Supermassive black hole $(3.932819 \mathrm{c} \mathrm{m} / \mathrm{s})$. This means that, the Aether speed on the surface of black holes are not conformed with the hierarchy of their masses as calculated according to Schwarzschild radius, viz. $r_{s}=$ $2 G M / c^{2}$. It is clear that the Schwarzschild radius is merely the Newtonian radius corresponding to a deflection angle of one radian. 
Thus, table II suggests reclassifying the category of Black Holes, according to value of the Aether speed $V_{e}$ at their surfaces, i.e. on the bases of equation (17), i.e. $r=4 G M / c V_{e}$.

\section{Doppler Effect in a Gravitational Field; the typical formula}

Now, let $r \rightarrow \infty$ in equation (7), then equation (7) will take the form;

$$
v^{\prime}=v e^{\frac{M G}{r^{\prime} c^{2}}}=v\left[1+\frac{M G}{r^{\prime} c^{2}}+\frac{1}{2 !}\left(\frac{M G}{r^{\prime} c^{2}}\right)^{2}+. .\right]
$$

This is the Doppler Effect in a gravitational field of mass $M$. Note that $v^{\prime}$ is the frequency of the wave in the gravitational field of the mass $M$ and $v$ is the frequency of the wave far away from the mass $M$, see figure $\mathrm{c}$. By considering only the first two terms of equation (18), then Doppler Effect in a gravitational field can be approximated by the following typical equation;

$$
v^{\prime} \cong v\left[1+\frac{M G}{r^{\prime} c^{2}}\right] \cong \frac{v}{\sqrt{1-\frac{2 M G}{r^{\prime} c^{2}}}}
$$

\section{Gravitational Time Dilation}

A clock in a gravitational field, of a non-rotating, an uncharged, spherically symmetric mass, runs more slowly according to the gravitational time dilation relationship derived from General Relativity, which is given by [4];

$$
T=\frac{T^{\prime}}{\sqrt{1-\frac{2 G M}{R c^{2}}}}
$$

This is distinct from the time dilation from the relative motion. In this equation $T^{\prime}$ is the time interval measured by a clock located in the gravitational field of the mass $M$, and $T$ is the time interval measured by a clock far away from the mass $M$. Equation (20) can be expressed into the following Binomial expansion,

$$
T=\frac{T^{\prime}}{\sqrt{1-\frac{2 G M}{R c^{2}}}}=T^{\prime}\left[1+\frac{M G}{R c^{2}}+\frac{1 \times 3}{2^{2} 2 !}\left(\frac{2 M G}{R c^{2}}\right)^{2}+\frac{1 \times 3 \times 5}{2^{3} 3 !}\left(\frac{2 M G}{R c^{2}}\right)^{3}+. .\right]
$$

Equations (18) and (21) clearly show the difference between the time dilation and Doppler Effect due to the Gravitational field of a mass $M$. Since we have the relation, $v=1 / T$, then the first two terms of equations (18) and (21) becomes identical. Hence the time dilation due to a gravity field of any mass $M$ is simply, becomes an approximation to Doppler Effect in the same gravity field of the mass $M$, or vice versa.

\section{The Experimental Verification of the vertical Aether flow}

Recently the vertical Michelson-Morley interferometer experiments have been conducted by Martin Grusenick in Germany (in August 14, 2009) [2], and by Frank G. Pearce in US (in Nov. 15, 2010) [3]. Each one of them constructs his own interferometer in his lab. After studying these experiments, they are considered a good experimental verification to the theory of the vertical Aether flow, although both Grusenik and Pearce did not attribute (or refer) their results (the oscillation of the interference pattern) to the vertical flow of Aether on Earth's surface. Grusenik introduced his experiment just as a new phenomenon never seen before and Pearce made the components of his interferometer from wood and refused the results of Grusenik, and considered it as induced strain effects in the aluminum components of Grusenik's interferometer, and these strain effects are due to the gravitational effect of the earth's field, which leads to these misleading results. However, reference [1] shows that both experiments lead to the same result and confirms this new discovered phenomenon of the vertical flow of Aether into Earth's surface as well as into any mass including fundamental building blocks (i.e. elementary particles).

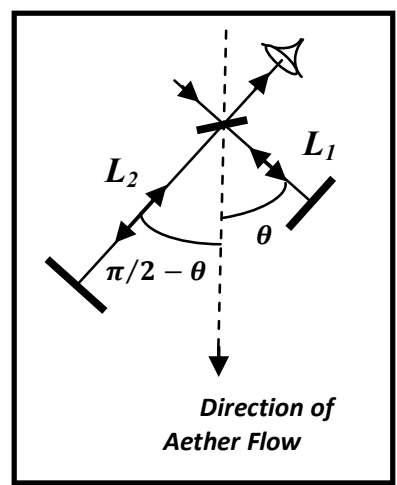

Figure e: Michelson-Morley interferometer at any arbitrary vertical position $\theta$. 
Referring to figure e, the difference between the two paths $\delta_{\text {ver }}$ will be given by [1];

$$
\delta_{\text {ver }}=2\left(L_{1}-L_{2}\right)+\left(\Delta_{1} \sin \theta-\Delta_{2} \cos \theta\right)
$$

Where; $L_{1}$ and $L_{2}$ are the arm lengths of Michelson-Morley interferometer, $\theta$ is the angle of the arbitrary vertical position and $\Delta_{1}, \Delta_{2}$ are the two curved paths of the light beams during their traveling journey between the two mirrors of the interferometer, which are given respectively by;

$$
\Delta_{1,2}=L_{1,2}^{3} / 3 R^{2}
$$

Where $\mathrm{R}$ is the earth's radius. Equation (22) represents the path difference in the vertical position of the interferometer, at any arbitrary position $\theta$. Equation (22) is a periodic function of $\theta$, which means that the interference pattern perform one complete oscillation with every complete cycle $(2 \pi)$ of $\theta$. Equation (22) is considered to be the first experimental proof for the theory of the vertical Aether flow. Note that; the difference between the two lengths of the arms of the interferometer in equation (22) can be adjusted to reduce the oscillation of the second term to a minimum, or to make it almost stationary. This pushed Pearce to believe that the oscillation of the pattern is an error due the gravitational effect on the metal parts of Grusenick interferometer.

\section{New Predictions; the Effect of Daily Earth Rotation about its axis on the Interference Pattern of the Vertical stationary Michelson-Morley Interferometer}

Reference [1] also shows that the oscillating interference pattern depends not only on the place of the interferometer but also on the daily time of the experiment, as given by the equation;

$$
\tau=\frac{2 L_{1}}{c} \frac{1}{1-\beta^{2}}-\frac{2 L_{2}}{c} \frac{1}{\sqrt{1-\beta^{2}}}
$$

Where $\tau$ is the time difference between the time taken by the journeys of the light beam to cut twice the arms of the interferometer $L_{1}$ and $L_{2}$, and $\beta=v / c$ is the speed ratio, where $v$ is the average resultant of the velocity of the earth during its daily trip around the sun, which is given by;

Where;

$$
v=V_{o}+(\omega R \cos \lambda) \cos \omega t
$$

$V_{o}$ : The average orbital speed of the earth around the sun.

$\omega:$ The earth angular speed.

$R$ : The earth radius.

$\lambda$ : The latitude of the place.

Equations (24) and (25) clearly show that the interference pattern will depend on the latitude $\lambda$ of the interferometer and will make a complete cycle (oscillation) every day due to the term $\cos \boldsymbol{\omega t}$, although the interferometer is kept stationary. This phenomenon is tested and confirmed in Germany by Martin Grusenick. Also the theory predicts that the interference pattern will not oscillate and will be stationary at the two poles of the Earth, where $\lambda=90^{\circ}$ which is a crucial difference between gravity and the concept of the vertical Aether flow. It is required to carry out this simple and extremely inexpensive experiment at North or South Pole to show that the interference pattern is stationary, which will confirm this new phenomenon. Equations (22) and (23) are considered to be the second experimental proof for the vertical Aether flow.

\section{Conclusion}

This paper shows that the vertical position of Michelson-Morley experiment is not the only possibility to explain the new phenomenon of the vertical Aether flow into any mass or any fundamental building block. The paper introduces three proofs for this new phenomenon. The third proof shows, for the first time, that the Cosmic Blue shift due to a gravitational field is a direct consequence of the vertical Aether flow into any mass. The vertical Aether speeds for different stellar objects have been given, which suggests a new classification for the categories of black holes. An alternative confirmation for this theory has been introduced through deriving new formulas for the Doppler Effect in a gravitational field. The paper shows the difference between the derived Doppler Effect and the time dilation in the gravitational field, as derived from the General Theory of Relativity, is a permissible approximation. The two theoretical expressions corresponding to the experimental results which verify the concept of the vertical Aether flow has been given. A new prediction has been proposed, for the first time, to confirm the theories presented in this paper.

The future extension of this new phenomenon to the quantum realm will enable us to establish the theory of everything, which unifies all the forces of nature in a very simple and logic way. In summary, this 
paper will radically change the way of our understanding of the universe and will enlighten our path to know the wisdom behind our destiny in this symphony of existence.

\section{References}

[1]. Isma'eel, M. E.: The Last Chapter of the Symphony of Existence $\left(^{\text {st }}\right.$ edition Publisher: Wahba, Egypt, $2010-2^{\text {nd }}$ edition, Publisher: Author House, UK, 2012).

http://www.amazon.com/dp/B00A8X5H16?tag=driyawijaya-20

http://www.amazon.com/The-Last-Chapter-Symphony-Existence/dp/1477234039\#reader 1477234039

http://www.amazon.com/The-Last-Chapter-Symphony-Existence/dp/1477234039\#

$\mathrm{http} / /$ www.google.com.eg/books?hl=en\&lr=\&id=8HtkDIOu9ykC\&oi=fnd\&pg=PĀ19\&dq $=$ cern + interpretation + of + interactions $+\%$ 2B + duck+\%2B +youtube\&ots $=$ ELa7jU1 z5W\&sig $=$ Hh6NVmtTZL x7zco-1UrX4HPnz9A\&redir esc $=\mathrm{y} \# \mathrm{v}=$ onepage \&q\&f $=$ false

[2]. http://www.youtube.com/watch? $=7 \mathrm{~T} 0 \mathrm{~d} 7 \mathrm{o} 8 \mathrm{X} 2-\mathrm{E} \&$ feature $=\mathrm{mfu}$ in_order\&list $=\mathrm{UL}$

[3]. http://www.youtube.com/watch? $\mathrm{v}=\mathrm{DH}-\mathrm{NC} 8 \mathrm{rvGvU} \& \mathrm{NR}=1$

[4]. http://en.wikipedia.org/wiki/Gravitational_time_dilation 